\title{
Cochlear Damage Changes the Distribution of Vesicular Glutamate Transporters Associated with Auditory and Nonauditory Inputs to the Cochlear Nucleus
}

\author{
Chunhua Zeng, ${ }^{1}$ Nishant Nannapaneni, ${ }^{1}$ Jianxun Zhou, ${ }^{1}$ Larry F. Hughes, ${ }^{2}$ and Susan Shore ${ }^{1}$ \\ ${ }^{1}$ Kresge Hearing Research Institute, Department of Otolaryngology, University of Michigan, Ann Arbor, Michigan 48109-0506, and ${ }^{2}$ Department of Surgery, \\ Division of Otolaryngology, Southern Illinois University School of Medicine, Springfield, Illinois 62794-9629
}

\begin{abstract}
Integration of multimodal information is essential for understanding complex environments. In the auditory system, multisensory integration first occurs in the cochlear nucleus (CN), where auditory nerve and somatosensory pathways converge (Shore, 2005). A unique feature of multisensory neurons is their propensity to receive cross-modal compensation after deafening. Based on our findings that the vesicular glutamate transporters, VGLUT1 and VGLUT2, are differentially associated with auditory nerve and somatosensory inputs to the CN, respectively (Zhou et al., 2007), we examined their relative distributions after unilateral deafening. After unilateral intracochlear injections of kanamycin (1 and 2 weeks), VGLUT1 immunoreactivity (ir) in the magnocellular CN ipsilateral to the cochlear damage was significantly decreased, whereas VGLUT2-ir in regions that receive nonauditory input was significantly increased 2 weeks after deafening. The pathway-specific amplification of VGLUT2 expression in the $\mathrm{CN}$ suggests that, in compensatory response to deafening, the nonauditory influence on $\mathrm{CN}$ is significantly enhanced. One undesirable consequence of enhanced glutamatergic inputs could be the increased spontaneous rates in $\mathrm{CN}$ neurons that occur after hearing loss and that have been proposed as correlates of the phantom auditory sensations commonly called tinnitus.
\end{abstract}

\section{Introduction}

Vesicular glutamate transporters (VGLUTs) package glutamate into synaptic vesicles and serve as excellent markers of glutamatergic projections. Although the homologous subtypes, VGLUT1 and VGLUT2, accumulate glutamate with similar transport kinetics, their distributions in the brain are distinct, with VGLUT1 more prominent in the cerebral and cerebellar cortices and hippocampus and VGLUT2 predominantly expressed in the thalamus, deep cerebellar nuclei, and brainstem regions including the cochlear nucleus (CN) (Fremeau et al., 2001; Herzog et al., 2001; Takamori et al., 2001; Kaneko et al., 2002).

In the CN, VGLUT1 and VGLUT2 are distinctly associated with synaptic terminals of auditory nerve fibers (ANF) and brainstem somatosensory nuclei, respectively. VGLUT1 expression is intense in the core region of ventral cochlear nucleus (VCN) and deep dorsal cochlear nucleus (DCN), where ANFs terminate on principal projection neurons, whereas VGLUT2 expression is stronger in the granule cell domain (GCD), which receives most of the nonauditory inputs to the CN (Wright and Ryugo, 1996; Ohlrogge et al., 2001; Zhou and Shore, 2004, 2006b; Haenggeli et

Received Jan. 9, 2009; revised Feb. 20, 2009; accepted Feb. 24, 2009.

This work was supported by National Institute on Deafness and Other Communication Disorders-National Institutes of Health Grant R01 DC DC004825 and Core Center Grant P30 DC-05188 and the Tinnitus Research Initiative. We thank Sanford Bledsoe for valuable comments on an earlier draft of this manuscript and Hersh Shroff and Clara Lee for excellent technical assistance.

Correspondence should be addressed to Susan Shore, Kresge Hearing Research Institute, Department of Otolaryngology, University of Michigan, 1301 East Ann Street, Ann Arbor, MI 48109-0506. E-mail: sushore@umich.edu. DOI:10.1523/JNEUROSCI.0208-09.2009

Copyright $\odot 2009$ Society for Neuroscience $\quad 0270-6474 / 09 / 294210-08 \$ 15.00 / 0$ al., 2005; Zhan et al., 2006; Zhan and Ryugo, 2007; Zhou et al., 2007). Furthermore, terminal endings originating in the spinal trigeminal nucleus (Sp5), cuneate nucleus, and lateral reticular nucleus colabel with VGLUT2 and not VGLUT1 (Zhou et al., 2007; Cui and Shore, 2008). In contrast, type I ANF terminals colabel with VGLUT1 but not VGLUT2. Type II ANFs (Benson and Brown, 2004) do not label with either VGLUT1 or VGLUT2 (Zhou et al., 2007). The VGLUT2-positive nonauditory endings activate granule cells, which project to the principal cells in the DCN, where they are combined with VGLUT1-positive ANF inputs, resulting in multisensory integration (Shore, 2005).

One feature unique to multisensory neurons is their propensity for receiving cross-modal compensation after sensory deprivation: In visually deprived mammals, auditory input from the inferior colliculus and auditory thalamus is redirected to the visual thalamus and cortex (Batzri-Izraeli et al., 1990; Izraeli et al., 2002; Piche et al., 2007). Similarly, the anterior ectosylvian region of the cat's parietal association cortex, which receives visual, auditory, and somatosensory inputs (Dehner et al., 2004; Meredith et al., 2006), becomes dominated by auditory and somatosensory inputs after visual deprivation and results in superior soundlocalizing abilities (Rauschecker, 1995). Deafness can also result in an infiltration of primary auditory cortex by neurons responding to somatosensory and visual stimuli (Hunt et al., 2006). Thus far, such cross-modal reorganization after deafness has not been demonstrated at peripheral levels of the auditory system. Here, we study cross-modal compensation in the $\mathrm{CN}$ by examining changes in the differential distributions of VGLUT isoforms associated with nonauditory (VGLUT2) and ANF (VGLUT1) pro- 
jections to the CN. Because VGLUT immunoreactivity (ir) is correlated with quantal release of glutamate (Wilson et al., 2005; Moechars et al., 2006), the degree of change in VGLUT-ir should be an indication of changes in the amount of glutamatergic transmission from auditory and nonauditory inputs.

\section{Materials and Methods}

Thirteen pigmented guinea pigs $(300-400$ g) with normal Preyer's reflexes were used in this study. Ten were unilaterally deafened and three were untreated controls. Five of the ten unilaterally deafened were allowed to survive for 1 week, and five were allowed to survive for 2 weeks after the deafening. For the unilaterally deafened animals, the contralateral and the ipsilateral CNs were compared with each other and to the normal controls $(N=6$, using both sides). All procedures were performed in accordance with the National Institutes of Health (NIH) Guidelines for the Use and Care of Laboratory Animals (NIH publication No. 80-23) and guidelines provided by the University Committee on Use and Care of Animals of the University of Michigan.

Deafening. The 10 animals to be unilaterally deafened were anesthetized with intramuscular injections of ketamine hydrochloride (Ketaset; $40 \mathrm{mg} / \mathrm{kg}$ ) and xylazine (Rompun; $10 \mathrm{mg} / \mathrm{kg}$ ) and placed in a stereotaxic frame (David Kopf Instruments). Rectal temperature was maintained at $38 \pm 0.5^{\circ} \mathrm{C}$ with a thermostatically controlled heating pad. Using a postauricular approach, a small incision was made behind the left ear and the skin retracted to expose the bulla. A small hole in the bulla was made and kanamycin $(20 \mu \mathrm{l}, 30 \%$ in normal saline) was injected into the cochlea using a Hamilton syringe, through the round window. After the injection, the skin overlying the bulla was sutured, and the animal was allowed to recover. After 1 week (five animals) or 2 weeks (five animals), auditory thresholds were tested using auditory brainstem responses (ABRs), and the animals were killed.

$A B R$ measurements. ABRs were performed in deafened animals before deafening, and 1 or 2 weeks after deafening on the day of killing. Normal animals received ABRs for both ears. ABRs were recorded in an electrically and acoustically shielded chamber (Acoustic Systems). Animals were anesthetized with $58.8 \mathrm{mg} / \mathrm{kg}$ ketamine, $2.4 \mathrm{mg} / \mathrm{kg}$ xylazine, and 1.2 $\mathrm{mg} / \mathrm{kg}$ acepromazine (Unit for Animal Laboratory Medicine, University of Michigan), and body temperature was maintained with thermostatically controlled heating pads. Subdermal recording electrodes were placed at vertex $(1 \mathrm{~cm}$ posterior to bregma), reference (ventral to the pinna on the tested ear), and ground (ventral to the pinna on the contralateral ear) sites.

Digital signals were generated and delivered to the Tucker Davis Technologies (TDT) hardware by a Pentium PC, using the TDT software package SigPlay32. Stimuli were generated using a sample rate of $100 \mathrm{kHz}$ at 16-bit resolution. Equalization to correct for the system response was performed on the digital waveforms in the frequency domain. Acoustic stimuli ( $15 \mathrm{~ms}$ tone bursts, with $1.5 \mathrm{~ms}$ rise/fall times), were presented at 10 per second. TDT System II hardware and SigGen/Biosig software (TDT) were used to present the stimulus and record responses. Tones (4, 10 , and $20 \mathrm{kHz}$ ) were delivered through a Beyer driver (Beyer Dynamic; aluminum-shielded enclosure made in house), using a speculum placed just inside the tragus. Up to 1024 responses were averaged for each stimulus level. Responses were collected for stimulus levels in $10 \mathrm{~dB}$ steps at higher stimulus levels, with additional $5 \mathrm{~dB}$ steps near threshold. Thresholds were interpolated between the lowest stimulus level where a response was observed and $5 \mathrm{~dB}$ lower where no response was observed.

After final ABR measurements, the animals were killed with Nembutal $(100 \mathrm{mg} / \mathrm{kg}$, i.p.) and transcardially perfused with $0.1 \mathrm{M}$ phosphatebuffered saline (PBS), followed by $4 \%$ paraformaldehyde in PBS. After perfusion fixation, the brain and cochleae were extracted and placed in the same fixative for $2 \mathrm{~h}$ at $4^{\circ} \mathrm{C}$. The brain was then transferred to $20 \%$ sucrose in $0.1 \mathrm{M} \mathrm{PBS}$ overnight at $4^{\circ} \mathrm{C}$. The brainstem was sectioned on a freezing microtome at a thickness of $40 \mu \mathrm{m}$. The cochleae were processed for hair cell counts (see below).

Cochlear hair cell and spiral ganglion assessments. Two animals (one at 1 week and one at 2 weeks after deafening) were processed for cochlear hair cell counts, and eight animals (four 1 week and four 2 week deaf- ened) were processed for spiral ganglion counts. Left (deafened) and right sides were compared.

Cochlear hair cell assessments. After the perfusions, the temporal bones were immediately removed, the round and oval windows exposed, and the cochleae fixed with intrascalar infusion of $4 \%$ paraformaldehyde in PBS through the round window. After fixation, cochleae were microdissected and stained with $1 \%$ rhodamine phalloidin. Surface preparations of the cochlear spiral were prepared, and individual turns of the organ of Corti were mounted on glass slides. Surface preparation assessment was performed under epifluorescent illumination on a Leitz photomicroscope. Surviving hair cells were counted in $0.19 \mathrm{~mm}$ reticules and plotted as cytocochleograms [percentage of outer hair cell (OHC) loss, relative to normal hearing ears] using a Microsoft Excel program kindly provided by Dr. David Moody (University of Michigan, Ann Arbor, MI). Raw data for each row of $\mathrm{OHC}$ were collected and assessed individually. In addition, data for the three rows of $\mathrm{OHC}$ were collapsed together and analyzed as a group.

Cell density of spiral ganglion cells. Every third midmodiolar section was mounted on a glass slide until 15 sections had been mounted and a random start point generated; the six sections from that point were used to generate an "analysis length" of $72 \mathrm{~lm}$. Six Rosenthal's canal profiles, visible on each section (two from the basal cochlea, two from the middle turn, and two from the apical turn), were assessed for each toluidine blue/basic fuchsin-stained section by an observer blind to treatment condition. Spiral ganglion cells were counted as normal if they met the following criteria: (1) had a diameter of 14-22 $\mu \mathrm{m}$ and an area of 153-380 $\mu \mathrm{m}^{2}$; (2) and had a nucleus, with a diameter of $5-10 \mu \mathrm{m}$. The outline of each profile of Rosenthal's canal was traced using a computer mouse. Cell count and cell area were then used to calculate cell density. All measurements were calculated using the MetaMorph Image Acquisition, Enhancement, and Analysis System (Molecular Devices).

Brain tissue and immunocytochemistry processing. Transverse sections of the brainstem were mounted in serial order on clean glass slides and air dried. Alternate serial sections were used to label VGLUT1 and VGLUT2.

All tissue processing was done at room temperature $\left(20-22^{\circ} \mathrm{C}\right)$ unless otherwise stated. The anti-VGLUT1 polyclonal antibody was generated in rabbits against Strep-Tag fusion protein containing amino acid residues 456-560 of rat VGLUT1 (Synaptic Systems; Cat. \#135 303), and the anti-VGLUT2 polyclonal antibody was generated in rabbits against Strep-Tag fusion protein containing amino acid residues $510-582$ of rat VGLUT2 (Synaptic Systems; Cat. \#135 403). Secondary antibodies were Alexa Fluor 488-conjugated goat anti-rabbit antibody (Invitrogen) and Cy3-conjugated goat anti-mouse antibody (Jackson Immunological Research Laboratories; code 115-165-146). All primary and secondary antibodies were diluted in the same blocking solution containing $1 \%$ normal goat serum (Jackson ImmunoResearch Laboratories; 005-000-121) in $0.1 \mathrm{M}$ phosphate buffer with $0.1 \%$ Triton X-100 (Sigma; 9002-93-1), $\mathrm{pH}$ 7.4. The sections were incubated in the blocking solution for $30 \mathrm{~min}$, followed by overnight incubation with primary antibodies (1:1000 for both VGLUT1 and VGLUT2). After thoroughly rinsing in PBS, sections were reacted for $2 \mathrm{~h}$ with the secondary antibody. Sections were then rinsed, dehydrated in graded ethanol, and coverslipped with Microcover (Micron). Negative controls were conducted on sections that were not treated with either primary or secondary antibodies, resulting in no immunolabeling. VGLUT antibodies were preincubated with corresponding synthetic peptides, resulting in negative immunolabeling. (Strep-Tag fusion protein containing amino acid residues 456-560 of rat VGLUT, Cat. \#135-3P, and Strep-Tag fusion protein containing amino acid residues 510-582 of rat VGLUT2, Cat. \#135-4P; Synaptic Systems). Western blots performed previously (Zhou et al., 2007) demonstrated anti-VGLUT1 antibody with single band at $\sim 60 \mathrm{kDa}$ on protein extracted from both CN and cerebellum and anti-VGLUT2 antibody as a single band at $\sim 65 \mathrm{kDa}$, corresponding to the molecular weights predicted for VGLUT1 and VGLUT2, respectively. Positive controls for VGLUT-ir were performed in the cerebellar cortex (Takamori et al., 2001; Kaneko et al., 2002; Hioki et al., 2003).

Image processing. Sections were examined using a fluorescent microscope equipped with the appropriate filters for rhodamine and Alexa Fluor 488 (Leica). Photomicrographs of immunolabeling were digitized 
and imported to MetaMorph for quantification of immunolabeling. The parameters for digitizing photomicrographs were determined in a pilot study with optimal contrast between labeling and background and were then kept constant across conditions and animals. Photomicrographs were imported to Adobe Photoshop for contrast adjustment that was consistent for all sections.

Analysis of VGLUT-ir was conducted for all subdivisions of the CN, with emphasis on the following regions: regions receiving primarily nonauditory inputs, i.e., the GCD, which includes the Shell region and the second layer of DCN, the fusiform cell layer (DCN2), and contains numerous small cells including granule cells (Weedman et al., 1996; Wright and Ryugo, 1996; Alibardi, 2004), and the deep DCN-DCN3, which contains some nonauditory terminals.

Regions that receive primarily type 1 ANF input include the anteroventral cochlear nucleus (AVCN) and posteroventral cochlear nucleus (PVCN) magnocellular areas and layer three of the DCN (AVCNm and PVCNm and DCN3). DCN1 (also called the molecular layer) is comprised mostly of the parallel fiber axons of granule cells but also contains some terminals from nonauditory regions (Zhou and Shore, 2004; Haenggeli et al., 2005).

Quantification and statistical analysis. Quantification was performed blind as to whether the tissue was from normal or deafened animals. The VGLUT-ir density was quantified in each of the following CN regions: DCN1, DCN2, and DCN3, the Shell region in the vicinity of the small cell cap, AVCNm, and PVCNm. The sections for AVCNm and PVCNm were taken from the midfrequency regions (i.e., halfway between the most dorsal aspect and the most ventral aspect of the VCN). This would, thereby, encompass only regions affected by the hearing loss (Fig. 1, high and midfrequency regions). For each side of each animal, three pictures $(40 \times)$ were taken at equal intervals from caudal to rostral for each of the above selected regions except DCN1 (i.e., one picture from the 25th percentile, one from the 50th percentile, and one from the 75th percentile). For DCN1, a higher magnification $(63 \times)$ was used to take three pictures evenly from caudal to rostral, leaving a total of 18 pictures per side in each animal. The photomicrographs were then transferred to MetaMorph for automatic quantification. To ensure that puncta were counted reliably, visual inspections and manual corrections were always conducted after each automated (thresholded) counting. This was to ensure that puncta were not merged by the thresholding procedure. The number of VGLUT-labeled puncta was divided by the chosen area to yield the puncta density. The chosen areas were consistent in size and position across animals. No distinction was made between puncta on cell bodies and puncta on dendrites or in the neuropil. Means and SEs of the mean were calculated for the puncta density of VGLUT1 and VGLUT2ir. The deafened and contralateral sides $(N=5,1$ week; $N=5,2$ weeks $)$ were compared with the normal controls $(N=6)$. A mixed effects, repeated-measures ANOVA was conducted with groups (normal, 1 week, 2 weeks) as a between-subjects factor and side (ipsilateral, contralateral), using the SPSS statistical package, region of the cochlear nucleus (DCN1, DCN2, DCN3, Shell, AVCN, PVCN), and section (caudal, medial, rostral) as within-subject factors.

\section{Results}

\section{Unilateral injections of kanamycin resulted in profound,} unilateral hearing loss

All 10 animals with unilateral intracochlear injections of kanamycin had profound, unilateral hearing losses as demonstrated with ABR measurements performed before killing (Fig. 2A). No $\mathrm{ABR}$ responses were measurable at $90 \mathrm{~dB}$ sound pressure level (the limits of our sound system) in the deafened ears. Figure $2 \mathrm{~A}$ shows the mean ABR thresholds for these animals at three test frequencies $(4,10$, and $20 \mathrm{kHz})$ for the left (ipsilateral, deafened) and right (contralateral) ears, as well as six normal control ears in three normal hearing animals.

Cytocochleograms show major inner and outer hair cell loss Figure $2 B$ shows an example of a typical cytocochleogram obtained for the ipsilateral and contralateral ear in one animal taken

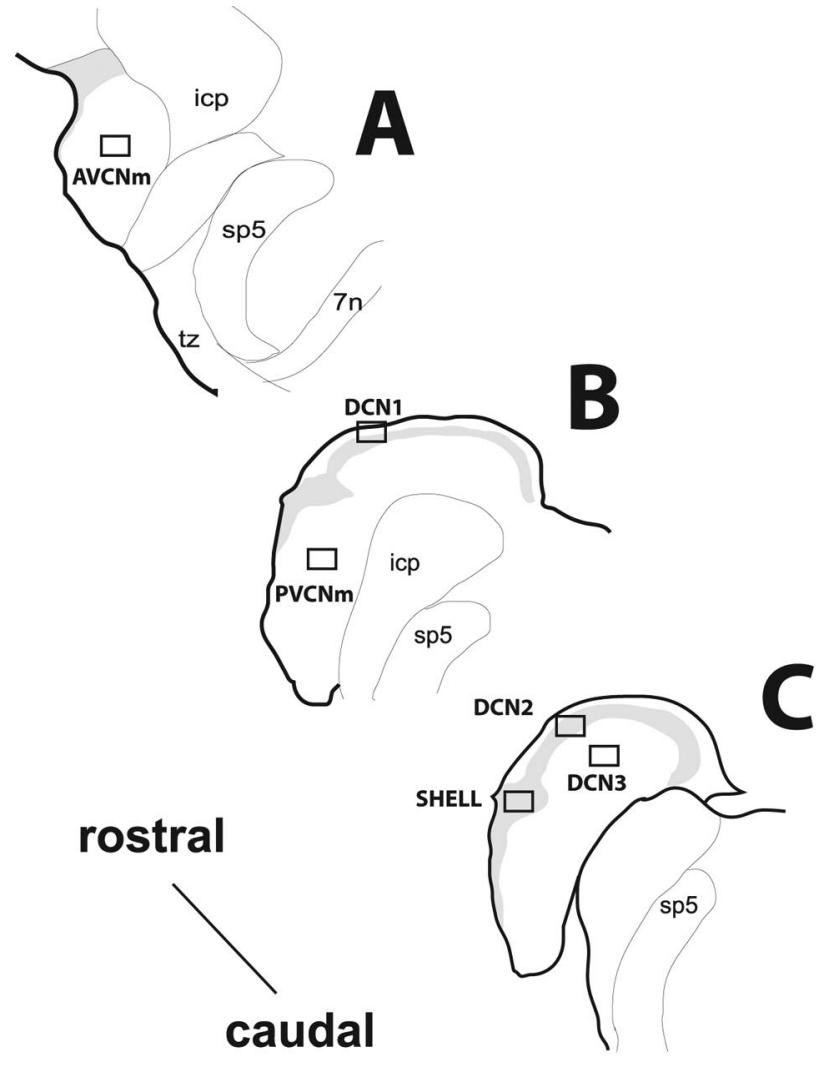

$1 \mathrm{~mm}$

Figure 1. Schematic of the locations within the $\mathrm{CN}$ that were used for measurements and micrographs. Each region of interest is indicated with square. $\boldsymbol{A}-\boldsymbol{C}$, Drawings of transverse sections from the rostral to caudal ends of CN. icp, Inferior cerebellar peduncle; tz, trapezoid body; $7 n$, facial nerve.

at 1 week after kanamycin injection. The cytocochleogram shows the percentage of missing inner and outer hair cells along the length of the cochlea. The deafened animal's ipsilateral cochlea (1 week) showed complete hair cell loss (inner and outer) for the basal half of the cochlea and severe inner and outer hair cell loss for the remaining $20 \%$ of the cochlea, excluding the apex. There was hair cell preservation in the apical $10 \%$ of the cochlea. This pattern of loss was also evident at 2 weeks after the injection. Such severe hair cell loss would be expected to result in the profound hearing losses exhibited using ABRs (Fig. 2A).

\section{Spiral ganglion density is significantly reduced 2 weeks after deafening}

Figure $2 C$ shows the mean spiral ganglion densities for the whole cochlea as well as the basal turn at 1 and 2 weeks after deafness. The left side for the whole cochlea $(F=221.24$; df $=1,28 ; p<$ $0.001)$ and the basal turn $(F=140.36$; $\mathrm{df}=1,40 ; p<0.001)$ shows decreased spiral ganglion density when compared with the right at both 1 and 2 weeks, additionally the decrease at 2 weeks is greater than at 1 week for the whole cochlea $(F=37.59$; $\mathrm{df}=1$, 28; $p<0.001)$ and the basal turn $(F=20.01$; df $=1,40 ; p<$ $0.001)$. Spiral ganglion density for the undeafened ear (right) was not significantly different between the 1 and 2 week groups ( $p>$ 0.719 for both whole and basal turn only) and is similar to values reported for normal animals (Miller et al., 2007). 
A

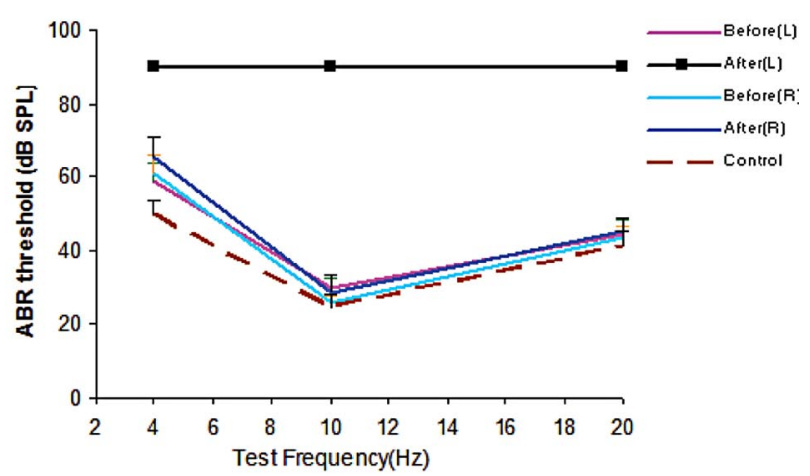

B

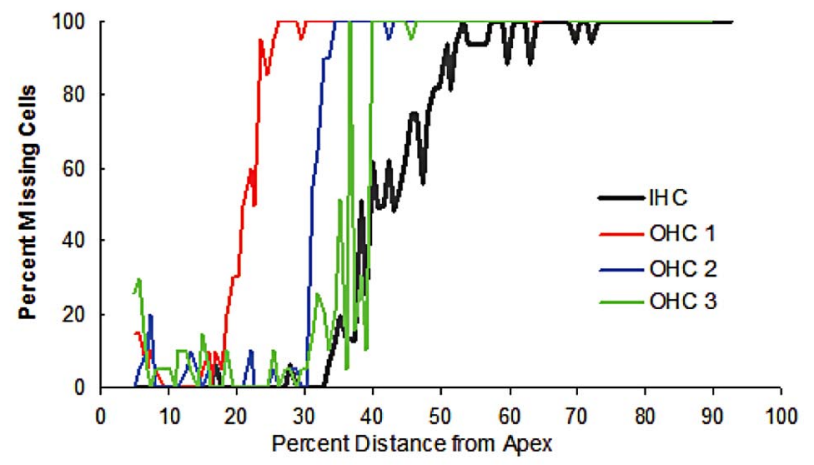

C

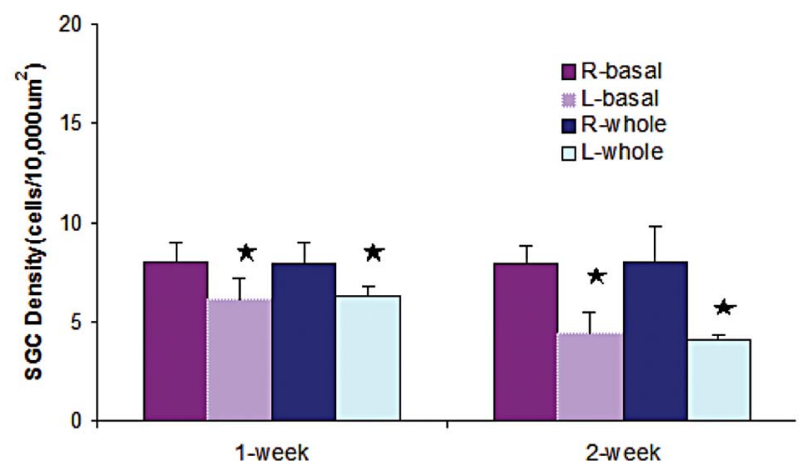

Figure 2. $A$, Mean ABR thresholds for five animals $1(N=5)$ and $2(N=4)$ weeks after deafening by an injection of kanamycin into the left cochlea. ABRs are shown before (left and right) and after (left and right) deafening. Three normal hearing, control animals (left and right ears) are also shown for comparison. ABRs indicate profound deafness at both 1 and 2 weeks after kanamycin injection. No effect of the kanamycin injections was observed in any of the right ears. Closed square symbols at $90 \mathrm{~dB}$ for 4 and $20 \mathrm{kHz}$ indicate no response at the limits of the speaker (indicated by upward arrows). $\boldsymbol{B}$, A representative cytocochleogram is shown from one guinea pig 1 week after a kanamycin injection into the left ear. The percentage of missing inner and outer hair cells is indicated along the length of the cochlea. The deafened cochlea showed complete hair cell loss (inner and outer) in the basal half of the cochlea and severe inner and outer hair cell loss in the remaining $20 \%$ of the cochlea, excluding the apex. There was hair cell preservation only in the apical $10 \%$ of the cochlea. The yellow line shows the extent of hair cell loss in the normal (contralateral) ear. C, Spiral ganglion counts $(\mathrm{SGC})$ are shown for eight animals, $1(N=4)$ and $2(N=4)$ weeks after kanamycin injections into the cochlea. Mean spiral ganglion densities for the whole cochlea and the basal turn at 1 and 2 weeks after cochlear damage are shown. Filled asterisks indicate significant differences of $p<0.01$.

\section{VGLUT expression is significantly altered in the cochlear} nuclei ipsilateral to unilateral deafening

VGLUT1-ir density, primarily representing VIIIth nerve synaptic terminals in $C N$, is decreased after unilateral deafness

Figure $3, A$ and $C$, shows photomicrographs of decreased VGLUT1-ir density in the ipsilateral AVCNm and PVCNm in one animal ( 2 weeks after deafening) compared with the con- tralateral side (Fig. $3 B, D$ ). The regions where the photomicrographs were taken are shown by arrows in Figure $1, A$ and $B$. The photomicrographs in Figure 3, $B$ and $D$, show strong VGLUT1 labeling in the contralateral AVCNm and PVCNm, especially around cell bodies in this region, reflecting VIIIth nerve input. This pattern is similar in the normal control animals (Zhou et al., 2007). In contrast, sparse labeling is observed on the side ipsilateral to the deafening (Fig. 3A,C). For the deafened animals, VGLUT1-ir density was significantly decreased in magnocellular cell areas of VCN (VCNm; AVCNm and PVCNm) that receive VIIIth nerve input on the ipsilateral side compared with both the contralateral side and the normal CNs at both 1 and 2 weeks after deafness (Fig. 4). A mixed effects, repeated-measures ANOVA was conducted with groups (normal, 1 week, 2 weeks) as a between-subjects factor and side (ipsilateral, contralateral), region of the cochlear nucleus (DCN1, DCN2, DCN3, Shell, AVCN, PVCN), and section (caudal, medial, rostral) as withinsubject factors. The main effects of side $(F=68.30 ; \mathrm{df}=1,13 ; p<$ $0.001)$ and region $(F=3605.28 ; \mathrm{df}=5,65 ; p<0.001)$ as well as the interactions of side with region $(F=64.47 ; \mathrm{df}=5,65 ; p<$ $0.001)$ and side with group $(F=18.34 ; \mathrm{df}=2,13 ; p<0.001)$ and side $\times$ region $\times$ group $(F=17.63 ; \mathrm{df}=10,65 ; p<0.001)$ were significant. The main effect of section was not significant, nor did it interact with any of the other factors indicating that the changes were relatively uniform across the rostral-caudal axis. The interaction of side with group and region was broken down into its simple effects, and Bonferroni adjusted comparisons were made as depicted in Figure 4. On the side, contralateral to the cochlear damage, there was no change in regulation of VGLUT1. On the side ipsilateral to the cochlear damage, no significant increase or decrease in VGLUT1 levels relative to normal is seen in the DCN2, DCN3, and Shell regions. However, the levels in the AVCN, PVCN, and DCN1 regions were significantly down for both 1 and 2 week groups after correction $(p<0.003)$ for those comparisons.

Note that DCN3 is also a recipient of nonauditory inputs (see below, "VGLUT2-ir, representing nonauditory synaptic terminals in CN (Zhou and Shore, 2006a), is increased after unilateral deafness").

\section{VGLUT2-ir, representing nonauditory synaptic terminals in $C N$}

(Zhou and Shore, 2006a), is increased after unilateral deafness

Figure 3 shows photomicrographs of increased VGLUT2-ir density in the ipsilateral Shell region of one animal (2 weeks after deafening) compared with the contralateral side (Fig. $3 E, F$ ). The region where the photomicrographs were taken is shown by an arrow in Figure $1 B$. Although VGLUT2 is intensely expressed in the Shell regions of all the animals, even on the contralateral side (Fig. 3F) as shown previously (Zhou et al., 2007), it is significantly increased above both contralateral and normal control levels at 2 weeks after deafening (Fig. 5). A mixed effects, repeated-measures ANOVA was conducted with groups (normal, 1 week, 2 weeks) as a between-subjects factor and side (ipsilateral, contralateral), region of the cochlear nucleus (DCN1, DCN2, DCN3, Shell, AVCN, PVCN), and section (caudal, medial, rostral) as within-subject factors. The main effects of side $(F=21.37 ; \mathrm{df}=1,13 ; p<0.001)$ and region $(F=151.18 ; \mathrm{df}=5$, $65 ; p<0.001)$ as well as the interactions of side with region $(F=$ $5.82 ; \mathrm{df}=5,65 ; p<0.001)$ and side with group $(F=6.56 ; \mathrm{df}=$ 2,$13 ; p=0.01$ ) were significant. Again, the main effect of section was not significant, nor did it interact with any of the other factors. The interaction of side with group and region was broken down into its simple effects, and Bonferroni adjusted compari- 
sons were made as depicted in Figure 5. On the side contralateral to the cochlear damage, there appears to be a temporary downregulation of VGLUT2 in the DCN2, DCN3, and Shell regions [after Bonferroni adjustment only DCN3 $(p<0.001)$ retained significance], which recovers to normal levels by 2 weeks. On the side ipsilateral to the cochlear damage, no significant increase or decrease in VGLUT2 levels is seen at 1 week; however, by week 2 , levels are significantly increased in the DCN2, DCN3, and Shell regions (all $p<$ 0.001 after correction). The increase in the AVCN region failed to retain significance after correction.

Thus, VGLUT2-ir density, primarily reflecting nonauditory inputs to the $\mathrm{CN}$, was thus significantly increased in regions previously shown to receive inputs from the Sp5, trigeminal ganglion, and other nonauditory nuclei (Weinberg and Rustioni, 1987; Shore et al., 2000; Zhou and Shore, 2004; Haenggeli et al., 2005; Shore and Zhou, 2006; Zhan et al., 2006). VGLUT2 puncta densities are significantly increased also in DCN3 ( $p<0.001)$, which receives both VIIIth nerve and nonauditory inputs. Interestingly, VGLUT2 is increased in ipsilateral AVCNm in the same region where VGLUT1 is decreased at 1 and 2 weeks after deafness, although this was not significant after Bonferroni correction.

Changes in the relative distributions of VGLUT1 and VGLUT2 across the CN To highlight the changes in the relative distributions of the two glutamate transporters, we replotted the data from Figures 4 and 5 for the deafened and normal ears as ratios. Figure 6 shows that, 2 weeks after deafening, the distributions of glutamatergic inputs become dominated by VGLUT2, signifying a major reorganization of projections from auditory nerve and nonauditory regions, including the spinal trigeminal nucleus, cuneate nucleus, and lateral reticular nucleus. Two weeks after deafness, there are decreases in VGLUT1 in all regions, but especially AVCNm and PVCNm, which receive major ANF inputs. In contrast, there are increases in VGLUT2 in DCN1, DCN2, the

Shell, and DCN3 (predominant recipients of nonauditory inputs), as well as AVCNm and PVCNm, suggesting a broadening of areas receiving nonauditory inputs.

\section{Discussion}

Glutamatergic projections from nonauditory nuclei to the $\mathrm{CN}$ terminate in $\mathrm{CN}$ regions that are distinct from those that receive auditory nerve synapses, i.e., primarily the Shell regions of $\mathrm{CN}$ and deep DCN (i.e., Shell, DCN2, DCN3) (Zhou and Shore, 2004; Haenggeli et al., 2005; Zhan et al., 2006; Zhou et al., 2007).
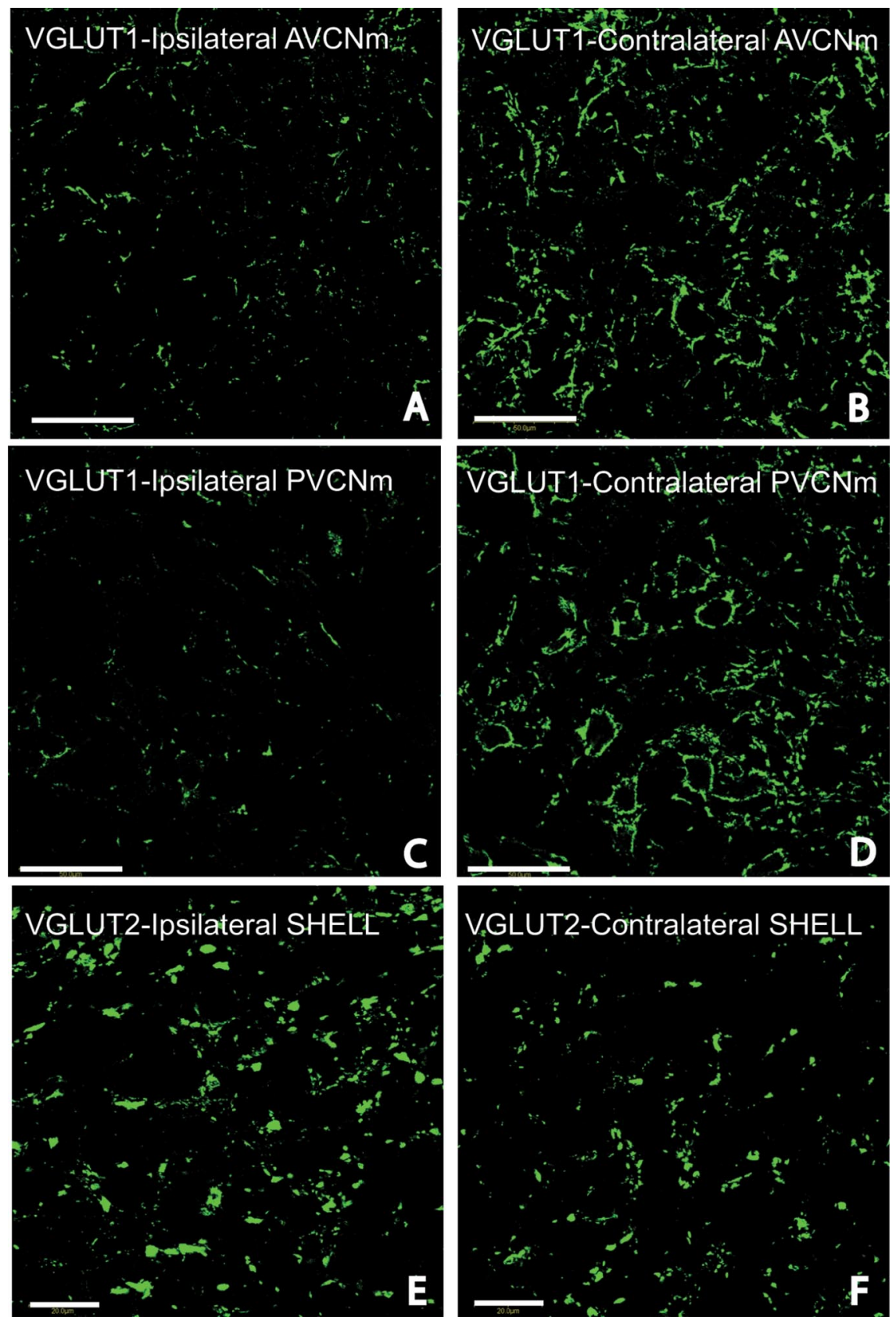

Figure 3. VGLUT1-ir is decreased, whereas VGLUT2 is increased in the CN ipsilateral to the cochlear damage. $A-D$, Photomicrographs of VGLUT1-ir from the VCNm of one animal (2 weeks after deafening). $\boldsymbol{A}, \boldsymbol{B}$, VGLUT1-ir in the AVCNm. VGLUT1 is strongly expressed in AVCNm on the control side (B) as previously shown (Zhou et al., 2007) but weakly expressed after deafening $(\boldsymbol{A})$. $\boldsymbol{C}$, D, VGLUT1-ir in the PVCNm. VGLUT1 is sparsely expressed in ipsilateral PVCNm compared with contralateral side. The decrease in VGLUT1-ir reflects primarily decreased VIIIth nerve synaptic inputs after kanamycin injections into the cochlea. $\boldsymbol{E}$, $\boldsymbol{F}$, Photomicrographs of VGLUT2-ir from SHELL regions of CN. VGLUT2-ir in the ipsilateral SHELL region is increased compared with the contralateral side. Scale bars: $\boldsymbol{A}-\boldsymbol{D}, 50 \mu \mathrm{m} ; \boldsymbol{E}, \boldsymbol{F}, 20 \mu \mathrm{m}$.

In contrast, type I VIIIth nerve fibers synapse on the cell bodies and dendrites of principal cells in the magnocellular regions of $\mathrm{CN}$ and some small cells in the cell cap (VCNm, DCNm) (Hackney et al., 1990; Snyder and Leake, 1997; Tsuji and Liberman, 1997). Type II ANFs project primarily to the dendrites of small neurons in the region of the small cell cap, but it is not yet clear whether they make synaptic contacts with granule cells (Benson and Brown, 2004), nor do they label with VGLUT1 or VGLUT2 (Zhou et al., 2007). Type II ANFs also project to cell bodies of multipolar or bushy cells, the targets of type I ANFs. 


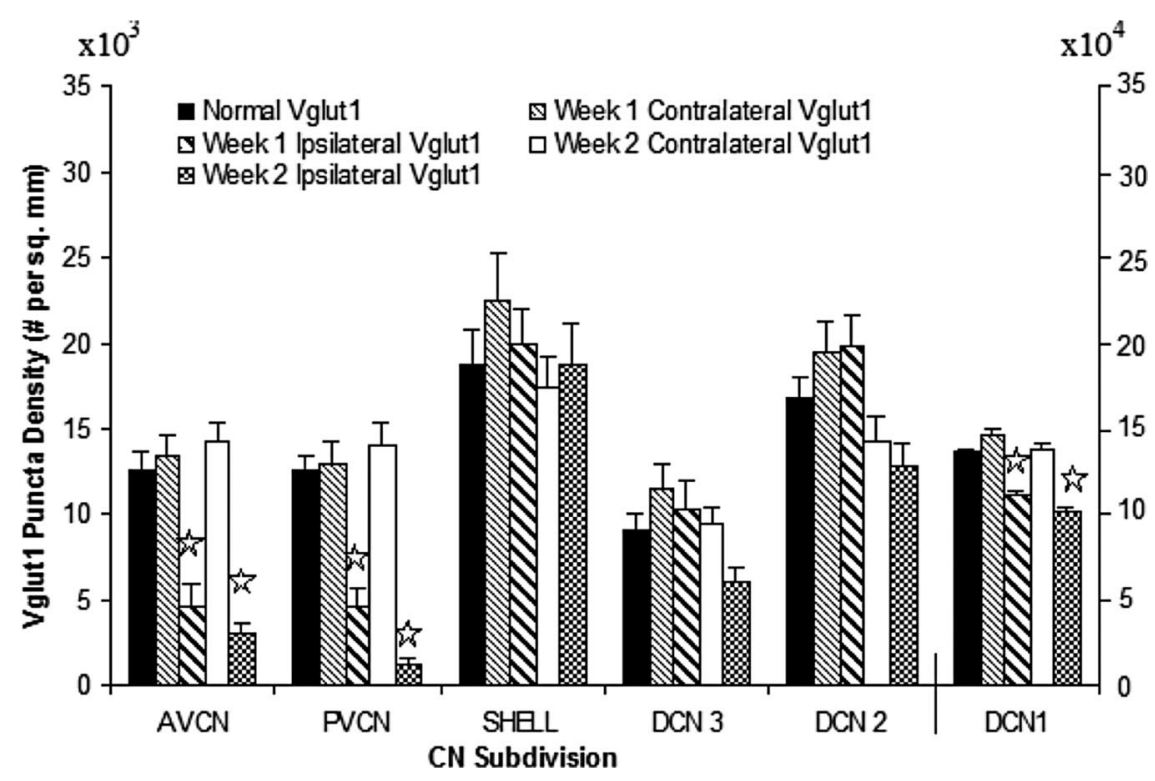

Figure 4. Mean VGLUT1 puncta densities in each CN subdivision in the ipsilateral, contralateral, and normal control CNs $(N=$ 6), $1(N=5)$ and 2 weeks ( $N=5)$ after deafness. VGLUT1 puncta densities (expressed as thousands per square millimeters) are significantly decreased in the ipsilateral (compared with the contralateral and normal CNs) AVCNm, PVCNm, and DCN1 compared with both the contralateral and normal control CNs ( $p<0.0003$, open stars). The magnocellular VCN receives major VIIIth nerve synaptic terminals.

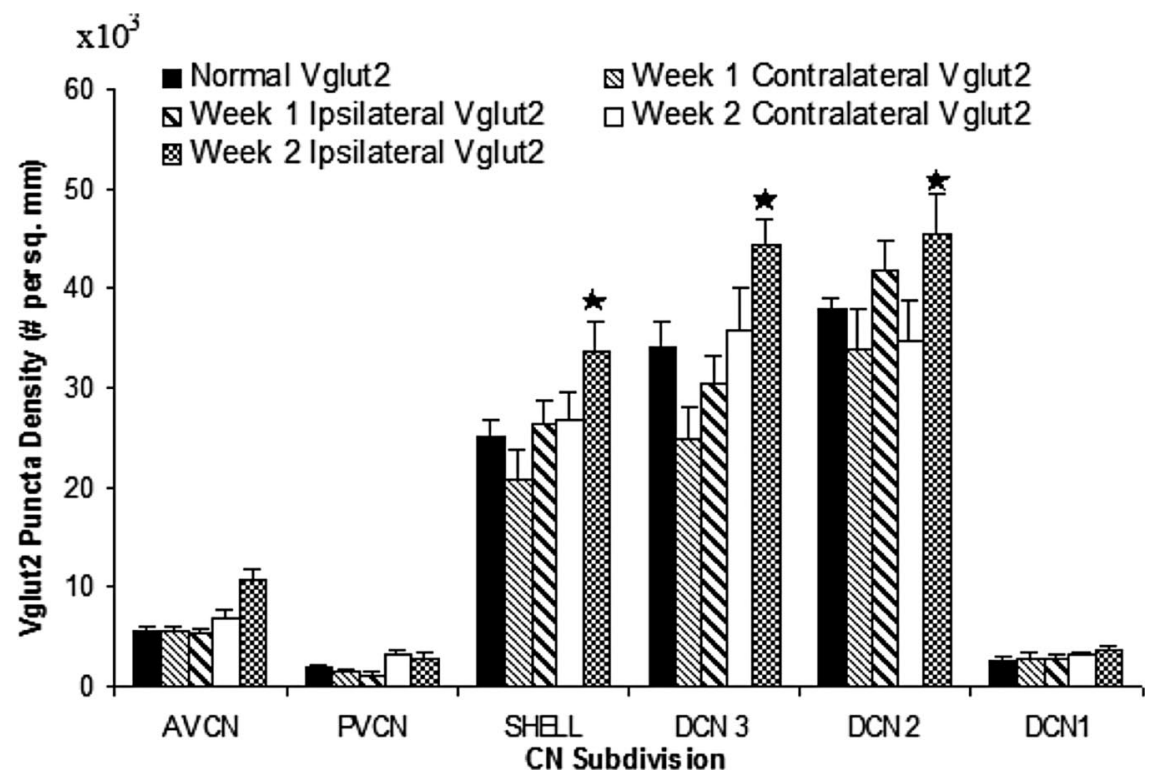

Figure 5. Mean VGLUT2 puncta densities in each CN subdivision, ipsilateral, contralateral, and normal control CNs $(N=6), 1$ $(N=5)$ and 2 weeks $(N=5)$ after deafness. VGLUT2 puncta densities at 2 weeks are significantly increased in the ipsilateral (compared with the contralateral and normal CNs) Shell and DCN2 that receive nonauditory synaptic terminals. Filled stars indicate $p<0.001$. VGLUT2 puncta densities are also significantly increased but to a lesser extent in DCN3, which receives both VIIIth nerve and nonauditory inputs. Error bars represent SEM.

Our observation that VGLUT1 is primarily (but not exclusively) associated with type I VIIIth nerve synapses, whereas VGLUT2 is associated almost exclusively with nonauditory inputs to the $\mathrm{CN}$ (Zhou et al., 2007), has enabled us to study changes in these inputs after cochlear damage. Unilateral cochlear kanamycin injections, which produced significant cochlear hair cell and spiral ganglion cell loss, accompanied by profound deafness, resulted in major, significant reductions in VGLUT1-ir in those regions receiving major VIIIth nerve input (AVCNm and PVCNm, with a similar trend in DCN3) 1 week and 2 weeks after deafness. Interestingly, there was no
VGLUT1-ir reduction in the Shell region, suggesting that the type I VIIIth nerve input to the Shell region (Tsuji and Liberman, 1997 ) is less affected by the cochlear damage. In agreement with our hypothesis that loss of VIIIth nerve fibers through deafening would cause cross-modal reinnervation of the cochlear nucleus, VGLUT2-ir was significantly increased in regions that receive nonauditory inputs (DCN2, DCN3, and Shell regions). These increases were increasingly evident at 2 weeks after deafness, suggesting the compensation is not immediate and may be the result of axonal sprouting (Boulland et al., 2007). This time line also correlates with the greater loss of spiral ganglion cells at 2 weeks after deafness.

Although it is well documented that cochlear deafferentation causes a decrease in the synaptic input from the VIIIth nerve to CN neurons (Muly et al., 2002; D'Sa et al., 2007), this is the first study to report potential increased synaptic inputs to the cochlear nucleus from nonauditory regions after cochlear damage. The increased VGLUT2-ir shown here could indicate an increase in the number of axonal inputs from nonauditory regions or simply an increase in the amount of glutamate transporter. The possibility that axonal sprouting may occur after cochlear damage has been suggested by reports of the reemergence of GAP-43 and synaptophysin after acoustic trauma (Illing and Horváth, 1995; Bilak et al., 1997; FuentesSantamaria et al., 2007). The present study provides evidence that some of these new axons are glutamatergic and originate in somatosensory nuclei, reticular formation, pontine nucleus, and other nonauditory areas (Shore et al., 2000; Zhou and Shore, 2004; Babalian, 2005; Haenggeli et al., 2005; Zhan and Ryugo, 2007; Zhou et al., 2007) to compensate for the loss of excitatory input from the cochlea. The signaling pathways needed to initiate these changes would likely be the parallel fibers, the axons of granule cells that carry the nonauditory information to the principal cells of the dorsal cochlear nucleus (Mugnaini et al., 1980; Manis, 1989). Indeed, the VGLUT1-ir parallel fibers in the present study (DCN1) showed less immunoreactivity after cochlear damage, possibly providing the needed signal for increasing axonal projections from nonauditory regions to their cell bodies, the granule cells. This region also showed an increase in VGLUT2-ir, probably reflecting an increase in the amount of nonauditory inputs that are occasionally seen in this region in the normal animal (Zhou and Shore, 2004; Haenggeli et al., 2005). Communication between the Shell region and VCN neurons has been suggested by the branching of the dendrites of D stellate cells into the granule cell domain (Oertel et al., 1990).

Over the past decade, the cochlear nucleus has been estab- 


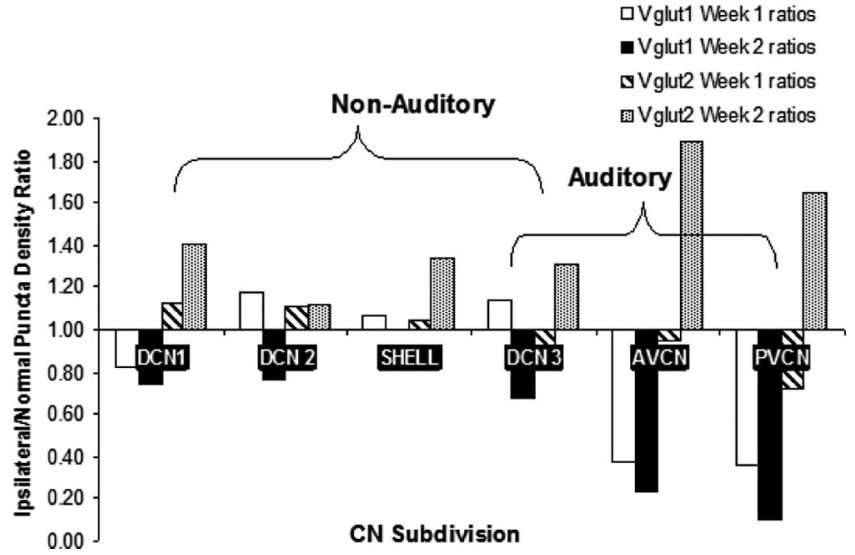

Figure 6. VGLUT immunoreactivity in the $C N$ ipsilateral $(N=10)$ to the deafening is replotted as ratios over the normal ears $(N=6)$, where $1=$ no difference between deafened and normal ears. $A$, The distributions of VGLUT1 and VGLUT2-ir across different regions of the $C N$ are shown in the same figure to highlight the changes in the relative distributions of the two VGLUT transporters after deafness. At both 1 and 2 weeks after deafness, ipsilateral VGLUT1 is decreased in VCNm (AVCN and PVCN) and DCN3, reflecting the large reduction of VIIIth nerve terminals after hair cell loss after the kanamycin injections. By 2 weeks, VGLUT1 is decreased more dramatically in all regions. In contrast, VGLUT2-ir is elevated above normal in DCN1, DCN2, and the Shell regions at 1 week after deafness and is elevated in all regions by 2 weeks. The areas receiving primarily auditory nerve inputs or nonauditory inputs are designated by the curly brackets above the graphs.

lished as a multisensory center that integrates auditory information provided by the VIIIth nerve with multimodal information from somatosensory and other nonauditory modalities to aid in orientation to sounds and suppression of body generated sounds (Davis et al., 1996; Kanold and Young, 2001; Shore et al., 2003, 2008; Shore, 2005). It is well known that cross-modal compensation can occur after deafferentation or sensory deprivation in one sense (Goel et al., 2006; Oh et al., 2007). Until now, this compensation has only been shown in higher structures, such as the inferior colliculus, superior colliculus, or cortex (Batzri-Izraeli et al., 1990; Izraeli et al., 2002; Piche et al., 2007). The present study demonstrates that cross-modal reinnervation can occur also in a second order structure, the CN. The changes demonstrated here are consistent with recent reports that, after cochlear damage, responses of $\mathrm{CN}$ neurons to trigeminal ganglion stimulation are enhanced as evidenced by shorter latencies and reduced thresholds, as well as a redistribution of excitatory and inhibitory responses (Shore et al., 2008). These changes could be adaptive, by enhancing the presumed functions of these pathways such as suppression of self-generated sounds, thus enabling the animal to attend more easily to external, environmental stimuli. Recent studies in primates indicate that the DCN circuitry documented for rodents and other mammals such as cats is also fully present in primates (Rubio et al., 2008), suggesting that these findings would be generalizable, even to humans.

One undesirable consequence of these increased glutamatergic inputs could be increased spontaneous rates in $\mathrm{CN}$ neurons that have been observed after hearing loss (Kaltenbach and Afman, 2000; Brozoski et al., 2002; Sumner et al., 2005; Shore et al., 2008), and that have been proposed as correlates of the phantom auditory sensations commonly called tinnitus (Zhang and Kaltenbach, 1998; Brozoski et al., 2002; Kaltenbach et al., 2004; Kaltenbach, 2007). In this context, the correlation of spiral ganglion loss with increases in VGLUT2 in the present study is consistent with the results of a recent study showing that loss of largediameter auditory nerve fibers can be a reliable predictor of tinnitus after acoustic exposure (Bauer et al., 2007).

\section{References}

Alibardi L (2004) Mossy fibers in granule cell areas of the rat dorsal cochlear nucleus from intrinsic and extrinsic origin innervate unipolar brush cell glomeruli. J Submicrosc Cytol Pathol 36:193-210.

Babalian AL (2005) Synaptic influences of pontine nuclei on cochlear nucleus cells. Exp Brain Res 167:451-457.

Batzri-Izraeli R, Kelly JB, Glendenning KK, Masterton RB, Wollberg Z (1990) Auditory cortex of the long-eared hedgehog (Hemiechinus auritus). I. Boundaries and frequency representation. Brain Behav Evol 36:237-248.

Bauer CA, Brozoski TJ, Myers K (2007) Primary afferent dendrite degeneration as a cause of tinnitus. J Neurosci Res 85:1489-1498.

Benson TE, Brown MC (2004) Postsynaptic targets of type II auditory nerve fibers in the cochlear nucleus. J Assoc Res Otolaryngol 5:111-125.

Bilak M, Kim J, Potashner SJ, Bohne BA, Morest DK (1997) New growth of axons in the cochlear nucleus of adult chinchillas after acoustic trauma. Exp Neurol 147:256-268.

Boulland JL, Ferhat L, Tallak Solbu T, Ferrand N, Chaudhry FA, StormMathisen J, Esclapez M (2007) Changes in vesicular transporters for gamma-aminobutyric acid and glutamate reveal vulnerability and reorganization of hippocampal neurons following pilocarpine-induced seizures. J Comp Neurol 503:466-485.

Brozoski TJ, Bauer CA, Caspary DM (2002) Elevated fusiform cell activity in the dorsal cochlear nucleus of chinchillas with psychophysical evidence of tinnitus. J Neurosci 22:2383-2390.

Cui Y, Shore S (2008) Topography of projections from the spinal trigeminal nucleus and lateral reticular formation in the guinea pig. Assoc Res Otolaryngol Abs 14:304.

Davis KA, Miller RL, Young ED (1996) Effects of somatosensory and parallel-fiber stimulation on neurons in dorsal cochlear nucleus. J Neurophysiol 76:3012-3024.

Dehner LR, Keniston LP, Clemo HR, Meredith MA (2004) Cross-modal circuitry between auditory and somatosensory areas of the cat anterior ectosylvian sulcal cortex: a 'new' inhibitory form of multisensory convergence. Cereb Cortex 14:387-403.

D'Sa C, Gross J, Francone VP, Morest DK (2007) Plasticity of synaptic endings in the cochlear nucleus following noise-induced hearing loss is facilitated in the adult FGF2 overexpressor mouse. Eur J Neurosci 26:666-680.

Fremeau RT Jr, Troyer MD, Pahner I, Nygaard GO, Tran CH, Reimer RJ, Bellocchio EE, Fortin D, Storm-Mathisen J, Edwards RH (2001) The expression of vesicular glutamate transporters defines two classes of excitatory synapse. Neuron 31:247-260.

Fuentes-Santamaría V, Alvarado JC, Henkel CK, Brunso-Bechtold JK (2007) Cochlear ablation in adult ferrets results in changes in insulin-like growth factor-1 and synaptophysin immunostaining in the cochlear nucleus. Neuroscience 148:1033-1047.

Goel A, Jiang B, Xu LW, Song L, Kirkwood A, Lee HK (2006) Cross-modal regulation of synaptic AMPA receptors in primary sensory cortices by visual experience. Nat Neurosci 9:1001-1003.

Hackney CM, Osen KK, Kolston J (1990) Anatomy of the cochlear nuclear complex of guinea pig. Anat Embryol (Berl) 182:123-149.

Haenggeli CA, Pongstaporn T, Doucet JR, Ryugo DK (2005) Projections from the spinal trigeminal nucleus to the cochlear nucleus in the rat. J Comp Neurol 484:191-205.

Herzog E, Bellenchi GC, Gras C, Bernard V, Ravassard P, Bedet C, Gasnier B, Giros B, El Mestikawy S (2001) The existence of a second vesicular glutamate transporter specifies subpopulations of glutamatergic neurons. J Neurosci 21:RC181.

Hioki H, Fujiyama F, Taki K, Tomioka R, Furuta T, Tamamaki N, Kaneko T (2003) Differential distribution of vesicular glutamate transporters in the rat cerebellar cortex. Neuroscience 117:1-6.

Hunt DL, Yamoah EN, Krubitzer L (2006) Multisensory plasticity in congenitally deaf mice: how are cortical areas functionally specified? Neuroscience 139:1507-1524.

Illing RB, Horváth M (1995) Re-emergence of GAP-43 in cochlear nucleus and superior olive following cochlear ablation in the rat. Neurosci Lett 194:9-12.

Izraeli R, Koay G, Lamish M, Heicklen-Klein AJ, Heffner HE, Heffner RS, Wollberg Z (2002) Cross-modal neuroplasticity in neonatally enucleated hamsters: structure, electrophysiology and behaviour. Eur J Neurosci 15:693-712. 
Kaltenbach JA (2007) The dorsal cochlear nucleus as a contributor to tinnitus: mechanisms underlying the induction of hyperactivity. Prog Brain Res 166:89-106.

Kaltenbach JA, Afman CE (2000) Hyperactivity in the dorsal cochlear nucleus after intense sound exposure and its resemblance to tone-evoked activity: a physiological model for tinnitus. Hear Res 140:165-172.

Kaltenbach JA, Zacharek MA, Zhang J, Frederick S (2004) Activity in the dorsal cochlear nucleus of hamsters previously tested for tinnitus following intense tone exposure. Neurosci Lett 355:121-125.

Kaneko T, Fujiyama F, Hioki H (2002) Immunohistochemical localization of candidates for vesicular glutamate transporters in the rat brain. J Comp Neurol 444:39-62.

Kanold PO, Young ED (2001) Proprioceptive information from the pinna provides somatosensory input to cat dorsal cochlear nucleus. J Neurosci 21:7848-7858.

Manis PB (1989) Responses to parallel fiber stimulation in the guinea pig dorsal cochlear nucleus in vitro. J Neurophysiol 61:149-161.

Meredith MA, Keniston LR, Dehner LR, Clemo HR (2006) Crossmodal projections from somatosensory area SIV to the auditory field of the anterior ectosylvian sulcus (FAES) in Cat: further evidence for subthreshold forms of multisensory processing. Exp Brain Res 172:472-484.

Miller JM, Le Prell CG, Prieskorn DM, Wys NL, Altschuler RA (2007) Delayed neurotrophin treatment following deafness rescues spiral ganglion cells from death and promotes regrowth of auditory nerve peripheral processes: effects of brain-derived neurotrophic factor and fibroblast growth factor. J Neurosci Res 85:1959-1969.

Moechars D, Weston MC, Leo S, Callaerts-Vegh Z, Goris I, Daneels G, Buist A, Cik M, van der Spek P, Kass S, Meert T, D’Hooge R, Rosenmund C, Hampson RM (2006) Vesicular glutamate transporter VGLUT2 expression levels control quantal size and neuropathic pain. J Neurosci 26:12055-12066.

Mugnaini E, Warr WB, Osen KK (1980) Distribution and light microscopic features of granule cells in the cochlear nuclei of cat, rat, and mouse. J Comp Neurol 191:581-606.

Muly SM, Gross JS, Morest DK, Potashner SJ (2002) Synaptophysin in the cochlear nucleus following acoustic trauma. Exp Neurol 177:202-221.

Oertel D, Wu SH, Garb MW, Dizack C (1990) Morphology and physiology of cells in slice preparations of the posteroventral cochlear nucleus of mice. J Comp Neurol 295:136-154.

Oh SH, Kim CS, Song JJ (2007) Gene expression and plasticity in the rat auditory cortex after bilateral cochlear ablation. Acta Otolaryngol 127:341-350.

Ohlrogge M, Doucet JR, Ryugo DK (2001) Projections of the pontine nuclei to the cochlear nucleus in rats. J Comp Neurol 436:290-303.

Piché M, Chabot N, Bronchti G, Miceli D, Lepore F, Guillemot JP (2007) Auditory responses in the visual cortex of neonatally enucleated rats. Neuroscience 145:1144-1156.

Rauschecker JP (1995) Developmental plasticity and memory. Behav Brain Res 66:7-12.

Rubio ME, Gudsnuk KA, Smith Y, Ryugo DK (2008) Revealing the molecular layer of the primate dorsal cochlear nucleus. Neuroscience 154:99-113.

Shore SE (2005) Multisensory integration in the dorsal cochlear nucleus: unit responses to acoustic and trigeminal ganglion stimulation. Eur J Neurosci 21:3334-3348.

Shore SE, Zhou J (2006) Somatosensory influence on the cochlear nucleus and beyond. Hear Res 216-217:90-99.

Shore SE, Vass Z, Wys NL, Altschuler RA (2000) Trigeminal ganglion innervates the auditory brainstem. J Comp Neurol 419:271-285.

Shore SE, El Kashlan H, Lu J (2003) Effects of trigeminal ganglion stimulation on unit activity of ventral cochlear nucleus neurons. Neuroscience 119:1085-1101.

Shore SE, Koehler S, Oldakowski M, Hughes LF, Syed S (2008) Dorsal cochlear nucleus responses to somatosensory stimulation are enhanced after noise-induced hearing loss. Eur J Neurosci 27:155-168.

Snyder RL, Leake PA (1997) Topography of spiral ganglion projections to cochlear nucleus during postnatal development in cats. J Comp Neurol 384:293-311.

Sumner CJ, Tucci DL, Shore SE (2005) Responses of ventral cochlear nucleus neurons to contralateral sound after conductive hearing loss. J Neurophysiol 94:4234-4243.

Takamori S, Rhee JS, Rosenmund C, Jahn R (2001) Identification of differentiation-associated brain-specific phosphate transporter as a second vesicular glutamate transporter (VGLUT2). J Neurosci 21:RC182.

Tsuji J, Liberman MC (1997) Intracellular labeling of auditory nerve fibers in guinea pig: central and peripheral projections. J Comp Neurol 381:188-202.

Weedman DL, Pongstaporn T, Ryugo DK (1996) Ultrastructural study of the granule cell domain of the cochlear nucleus in rats: mossy fiber endings and their targets. J Comp Neurol 369:345-360.

Weinberg RJ, Rustioni A (1987) A cuneocochlear pathway in the rat. Neuroscience 20:209-219.

Wilson NR, Kang J, Hueske EV, Leung T, Varoqui H, Murnick JG, Erickson JD, Liu G (2005) Presynaptic regulation of quantal size by the vesicular glutamate transporter VGLUT1. J Neurosci 25:6221-6234.

Wright DD, Ryugo DK (1996) Mossy fiber projections from the cuneate nucleus to the cochlear nucleus in the rat. J Comp Neurol 365:159-172.

Zhan X, Ryugo DK (2007) Projections of the lateral reticular nucleus to the cochlear nucleus in rats. J Comp Neurol 504:583-598.

Zhan X, Pongstaporn T, Ryugo DK (2006) Projections of the second cervical dorsal root ganglion to the cochlear nucleus in rats. J Comp Neurol 496:335-348.

Zhang JS, Kaltenbach JA (1998) Increases in spontaneous activity in the dorsal cochlear nucleus of the rat following exposure to high-intensity sound. Neurosci Lett 250:197-200.

Zhou J, Shore S (2004) Projections from the trigeminal nuclear complex to the cochlear nuclei: a retrograde and anterograde tracing study in the guinea pig. J Neurosci Res 78:901-907.

Zhou J, Shore S (2006a) Changes in the distribution of vesicular glutamate transporters in the cochlear nucleus after deafness: relationship to auditory andnon-auditory inputs. Assoc Res Otolaryngol Abs 668.

Zhou J, Shore S (2006b) Convergence of spinal trigeminal and cochlear nucleus projections in the inferior colliculus of the guinea pig. J Comp Neurol 495:100-112.

Zhou J, Nannapaneni N, Shore S (2007) Vessicular glutamate transporters 1 and 2 are differentially associated with auditory nerve and spinal trigeminal inputs to the cochlear nucleus. J Comp Neurol 500:777-787. 